\title{
Study on Properties of Branched Hydrophobically Modified Polyacrylamide for Polymer Flooding
}

\author{
Lei-Ting Shi, ${ }^{1}$ Cheng Li, ${ }^{2}$ Shan-Shan Zhu, ${ }^{1}$ Jie Xu, ${ }^{2}$ Bao-Zong Sun, ${ }^{3}$ and Zhong-Bin Ye ${ }^{1}$ \\ ${ }^{1}$ State Key Laboratory of Oil \& Gas Reservoir and Exploitation Engineering, Southwest Petroleum Institute, Chengdu 610500, China \\ ${ }^{2}$ College of Chemistry \& Chemical Engineering, Southwest Petroleum University, Chengdu 610500, China \\ ${ }^{3}$ Institute of Xinjiang Oilfield Company, Karamayi, Xinjiang 834000, China
}

Correspondence should be addressed to Cheng Li; 343317905@qq.com

Received 4 April 2013; Accepted 19 August 2013

Academic Editor: Ibnelwaleed Ali Hussien

Copyright (C) 2013 Lei-Ting Shi et al. This is an open access article distributed under the Creative Commons Attribution License, which permits unrestricted use, distribution, and reproduction in any medium, provided the original work is properly cited.

\begin{abstract}
The effect of partially hydrolyzed polyacrylamides (HPAMs) used for polymer flooding is unsatisfactory under the conditions of high temperature and high salinity. In order to improve the viscosifying ability of HPAM, branched macromolecular skeleton monomer is used to change the linear backbone structure. A new branched hydrophobically modified polyacrylamide (BHMPAM) was synthesized by the free radical copolymerization of functionalized branched macromolecular skeleton monomer, acrylamide $(\mathrm{AM})$, acrylic acid (AA), and hydrophobic monomer hexadecyl-allyl-dimethyl ammonium chloride ( ${ }_{16}$ DMAAC). The properties of polymer solution were characterized; the results of the experiments showed that BHMPAM exhibited the properties of pseudoplastic fluid, and the viscosity of BHMPAM was $345.9 \mathrm{mPa} \cdot \mathrm{s}$ (polymer concentration was $1750 \mathrm{mg} / \mathrm{L}$ ) under the condition of $75^{\circ} \mathrm{C}$ and $9374 \mathrm{mg} / \mathrm{L}$ of salinity. Moreover, BHMPAM also performed well in viscoelasticity which can meet the property requirements for EOR polymer.
\end{abstract}

\section{Introduction}

Currently, the common used oil displacement agent is partially hydrolyzed polyacrylamide (HPAM), the viscosity of which, however, descends drastically with the increase of temperature and salinity [1]. Hence, along with the continuous development of oilfield and with the increasing severity of reservoir conditions, polyacrylamide should be improved in molecular weight or by increasing polymer concentration to satisfy the necessary viscosity. The former way makes its antishearing capacity decrease, while the latter makes its production costs increase dramatically. Hydrophobically associating water-soluble polymers (HAWSP), which contain a small amount of hydrophobic groups on the macromolecular chains, are developed to solve the problem of PAM as an oil displacement agent [2-4]; the viscosifying ability of HAWSP is improved, but HAWSP performs poorly in shear tolerance due to its linear molecular chain [5].

Compared with the traditional linear polymer, branched macromolecule, as a new highly branched polymer whose structure is similar to three-dimensional sphere, owns its unique features, including shear tolerance, rheology, and thermal stability [6, 7]. Moreover, combining branched macromolecule with linear hydrophobically associating polymer can improve the anti-shearing performance, temperature tolerance, and salt tolerance of the polymer aqueous solution, because there are more combination points producing hydrophobic intramolecular interaction, leading to stronger network structure in solution. In order to improve traditional polyacrylamide's properties and its applications in high temperature and salinity reservoirs, HPAM should be modified to change the viscosifying way. In this paper, we synthesized a new branched hydrophobically modified polyacrylamide (BHMPAM) and evaluated the properties of the polymer solution.

\section{Experiments Involved}

2.1. Materials. Ethylene diamine ( $\geq 99.0 \%)$, methyl acrylate $(\geq 99.0 \%)$, methanol $(\geq 99.0 \%)$, sulfuric acid (95-98\%), maleic anhydride $(\geq 99.0 \%)$, acrylic acid $(\geq 99.0 \%)$, dimethyl 
sulfoxide, acrylamide $(\geq 99.0 \%)$, sodium hydrogen sulfite $(\geq 58.5 \%)$, and ammonium persulfate $(\geq 98.0 \%)$ were purchased from Chengdu Kelong Chemical Reagent Factory. $\mathrm{C}_{16}$ DMAAC is laboratory homemade. HPAMs (viscosity average molecular weight is $1.72 \times 10^{7}$, and degree of hydrolysis is 15\%) were purchased from French SNF Company. The experiment water was prepared with doubly distilled water, and the ionic composition was shown in Table 1.

\subsection{Synthesis}

2.2.1. Synthesis and Functionalization of Branched Polyamidoamine. The methods of synthesis and modification of branched polyamidoamine macromolecular skeleton monomer refer to the literature [8]. The processes can be described as follows. At first, certain amounts of ethylene diamine and methyl acrylate were added into a three-necked flask with methanol as solvent under nitrogen at room temperature for $48 \mathrm{~h}$. Then, the mixture was put on the rotary evaporator to react several hours under different temperatures, and the branched polyamidoamine was prepared. A certain quantity of branched polyamidoamine was dissolved with dimethyl sulfoxide as solvent in the wide-necked bottle. A certain quantity of maleic anhydride was added slowly when branched polyamidoamine was dissolved completely in order to obtain modified branched polyamidoamine macromolecule.

2.2.2. Synthesis of BHMPAM. The mechanical stirrer and $\mathrm{N}_{2}-$ inlet/outlet installation were equipped in a $250 \mathrm{~mL}$, threeneck round-bottomed flask, in which acrylamide and acrylic acid were added at the mass ratio of $4: 1$ with distilled water as solvent, and then a certain quantity of hydrophobic monomer and functionalized branched macromolecular skeleton monomer was added. The initiators (sodium bisulfite and ammonium persulfate) were added at $45^{\circ} \mathrm{C}$, and then solution reacted for $12 \mathrm{~h}$. After reaction, the products were purified by methanol several times and then dried for several days under vacuum at $50^{\circ} \mathrm{C}$, so BHMPAM were obtained. The result of measuring BHMPAM's viscosity average molecular weight was $7 \times 10^{6}$. The structure of BHMPAM compared to HPAM was shown in Figure 1.

2.3. Polymer Dissolving Time Test. Referring to China's National Standards about determination of the dissolution rate of powdered polyacrylamide (GB12005.8-1989), dissolving time was conducted on a DDS-11A conductivity meter at (30 $0.1)^{\circ} \mathrm{C}$. The solvents used were distilled water and synthetic formation water.

2.4. Polymer Dissolution Preparation. For the dissolution of the polymers, a stock solution of $5000 \mathrm{mg} / \mathrm{L}$ was prepared by stirring the synthetic formation water (or distilled water) with a mechanical stirrer until a vortex was established. The polymer powder was poured slowly into the vortex and stirred for at least $3 \mathrm{~h}$. This stock solution was subsequently diluted to obtain the desired concentration.
2.5. Viscosity Measurements. All viscosities were measured at a shearing rate of $7.34 \mathrm{~s}^{-1}$ with Brookfield DV-III at $65^{\circ} \mathrm{C}$.

2.6. Rheological Property Test. Rheological measurements were conducted on a HAAKE RS600 Rotational Rheometer (Germany) at $65^{\circ} \mathrm{C}$. Shear viscosity range was $0.01-200 \mathrm{~s}^{-1}$.

2.7. Viscoelasticity Test. Storage modulus $\left(G^{\prime}\right)$ and loss modulus $\left(G^{\prime \prime}\right)$ measurements were conducted on a HAAKE RS600 Rotational Rheometer (Germany) at $65^{\circ} \mathrm{C}$. Immobilization stress is $0.1 \mathrm{~Pa}$, and vibration frequency range is $0.1-1.0 \mathrm{~Hz}$.

\section{Results and Discussion}

3.1. Polymer Solubility. The results of dissolving time are shown in Table 2.

As shown in Table 2, BHMPAM exhibits good solubility in distilled water and in synthetic formation water. Normally, in the laboratory study, a dissolving time of polymer of less than 6 hours can be applied in the engineering field, and the time changes with the dissolving process conditions in engineering field. Although BHMPAM's solubility was poorer than HPAM's, the dissolving time of BHMPAM could still meet the solubility requirements in engineering application. The hydrophobic groups contained in BHMPAM were difficult to dissolve in the polar aqueous solution, and the strong molecular structure slowed down the diffusion rate between the polymer molecules and the solvent molecules, so that the BHMPAM's dissolving time was longer.

\subsection{Effect of Polymer Solution Concentration on Apparent} Viscosity. The viscosity of BHMPAM increased swiftly with the increasing polymer concentration when the polymer concentration was between 1000 and $1500 \mathrm{mg} / \mathrm{L}$, as shown in Figure 2. BHMPAM exhibited the unique viscosifying ability, which meant that BHMPAM could substantially improve the mobility control capacity of aqueous phase in lower polymer concentrations.

The viscosity of polymer was constituted by the structural viscosity and bulk viscosity [9]. The structural viscosity was influenced by the structural strength of aggregates which was formed by polymer molecular chain in solution, and the key factor of affecting bulk viscosity was molecular weight. Due to intermolecular association and high degree of branching increasing the intermolecular entanglement, the structural viscosity was enhanced, so that the viscosity of BHMPAM is rather high while the molecular weight of BHMPAM was less than HPAM.

3.3. Effect of Temperature on Apparent Viscosity. In general, the viscosity of polymer solutions shows a temperature dependent behavior. As shown in Figure 3, the viscosity of BHMPAM changed little with the temperature below $55^{\circ} \mathrm{C}$ and then decreased with the increasing temperature. The viscosity of BHMPAM decreased by $43.4 \%$. For HPAM, the viscosity was plummeting quickly at the elevated temperature, and the viscosity decreased by $63.5 \%$. Hence, it could be shown that the BHMPAM performed better at temperature 
TABLE 1: The ionic composition of synthetic formation water.

\begin{tabular}{lccccccccc}
\hline \multirow{2}{*}{ Synthetic formation water } & Composition & $\mathrm{Na}^{+}$and $\mathrm{K}^{+}$ & $\mathrm{Ca}^{2+}$ & $\mathrm{Mg}^{2+}$ & $\mathrm{CO}_{3}{ }^{2-}$ & $\mathrm{HCO}_{3}^{-}$ & $\mathrm{SO}_{4}{ }^{2-}$ & $\mathrm{Cl}^{-}$ & $\mathrm{TDS}^{-}$ \\
& Content $(\mathrm{mg} / \mathrm{L})$ & 3091.96 & 276.17 & 158.68 & 14.21 & 311.48 & 85.29 & 5436.34 & 9374.12 \\
\hline
\end{tabular}

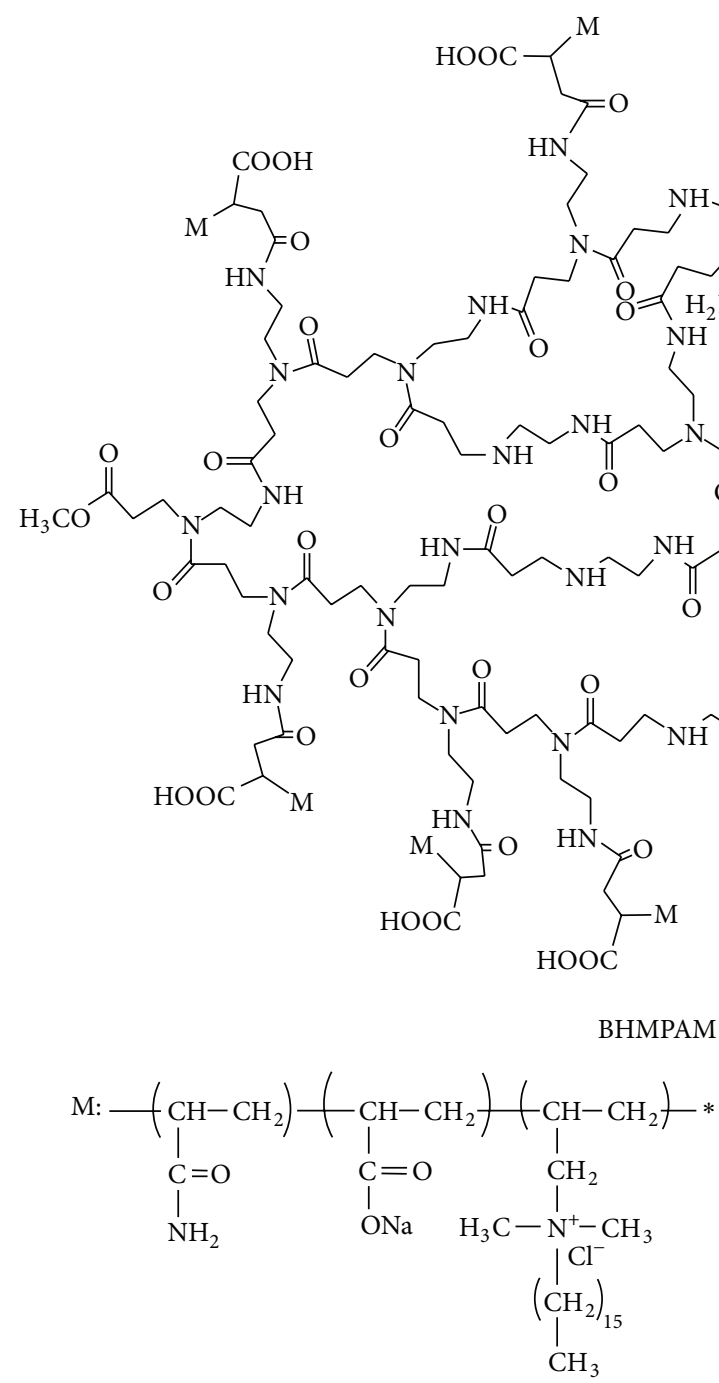

(a)

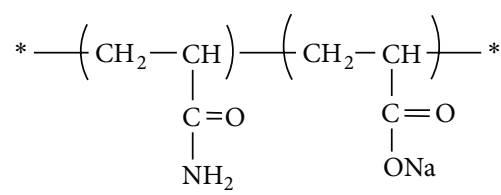

HPAM

(b)

FIGURE 1: The structure of BHMPAM compared to HPAM.

tolerance. When BHMPAM was used in high temperature reservoirs, it could also meet the viscosifying requirement.

The influence of temperature on the viscosity of BHMPAM appeared in two aspects $[10,11]$. On one hand, the increasing temperature intensified the thermal motion of the hydrophobic groups and water molecules, which changed the hydration around the hydrophobic group and weakened the hydrophobic interaction, so the viscosity decreased. On the other hand, the intermolecular association increased with the rising temperature, because the hydrophobic association was an endothermic entropy-driven process. As a result of the two aspects, the viscosity of BHMPAM decreased by $12.6 \%$ below $55^{\circ} \mathrm{C}$ and decreased by $47.6 \%$ when temperature reached $75^{\circ} \mathrm{C}$. But for HPAM, the hydrogen bond interactions among molecules decreased with the rise of temperature, which curled the molecular chains, so the viscosity decreased quickly over the whole temperature range.

3.4. Effect of Shearing Rate on Apparent Viscosity. As shown in Figure 4, BHMPAM exhibited good shearing thinning under the high shearing rate; when the shearing rate is low, BHMPAM solution presented high viscosity. In polymer flooding, the shearing rate was higher in near borehole zones, BHMPAM's shearing thinning under high shearing rate is beneficial for its injection, the shear rate decreased after solution was injected into formation, and the viscosity of BHMPAM was high under low shearing rate which made it perform well in the mobility control capacity. 
TABLE 2: The solubility of BHMPAM and HPAM.

\begin{tabular}{lccccc}
\hline Sample & Granularity $(\mu \mathrm{m})$ & Stirring rate $(\mathrm{r} / \mathrm{min})$ & Temperature $\left({ }^{\circ} \mathrm{C}\right)$ & Concentration $(\mathrm{mg} / \mathrm{L})$ & Time $(\mathrm{h})$ \\
\hline BHMPAM $^{1}$ & $350 \sim 833$ & 90 & 30 & 5000 & 2.5 \\
BHMPAM $^{2}$ & $350 \sim 833$ & 90 & 30 & 5000 & 4.3 \\
HPAM $^{1}$ & $350 \sim 833$ & 90 & 30 & 5000 \\
HPAM $^{2}$ & $350 \sim 833$ & 90 & 30 & 5000 & 1.6 \\
\hline
\end{tabular}

(1: the solvent is distilled water; 2 : the solvent is synthetic formation water.)

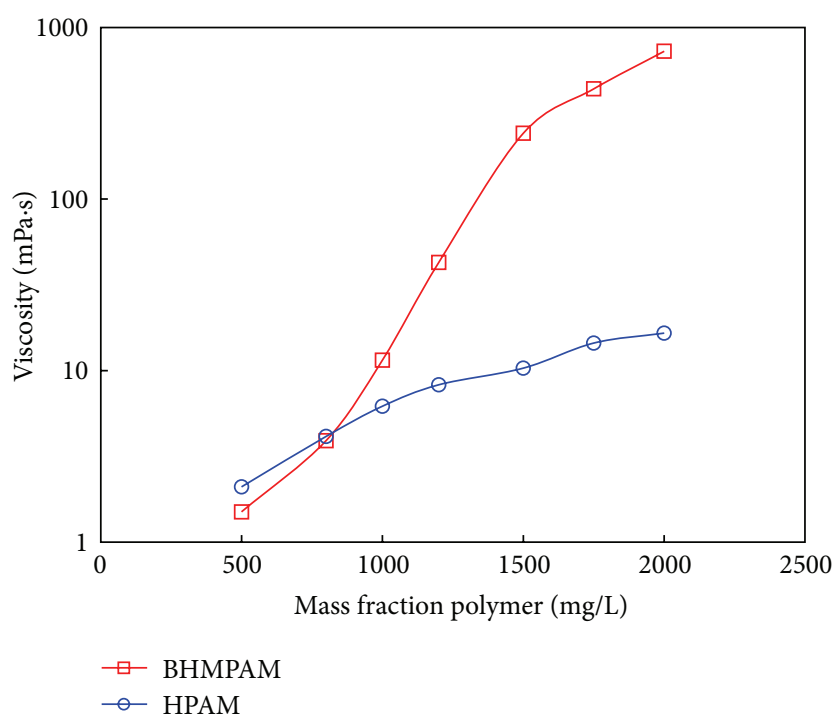

FIGURE 2: Viscosity versus polymer concentration of BHMPAM compared to HPAM in synthetic formation water.

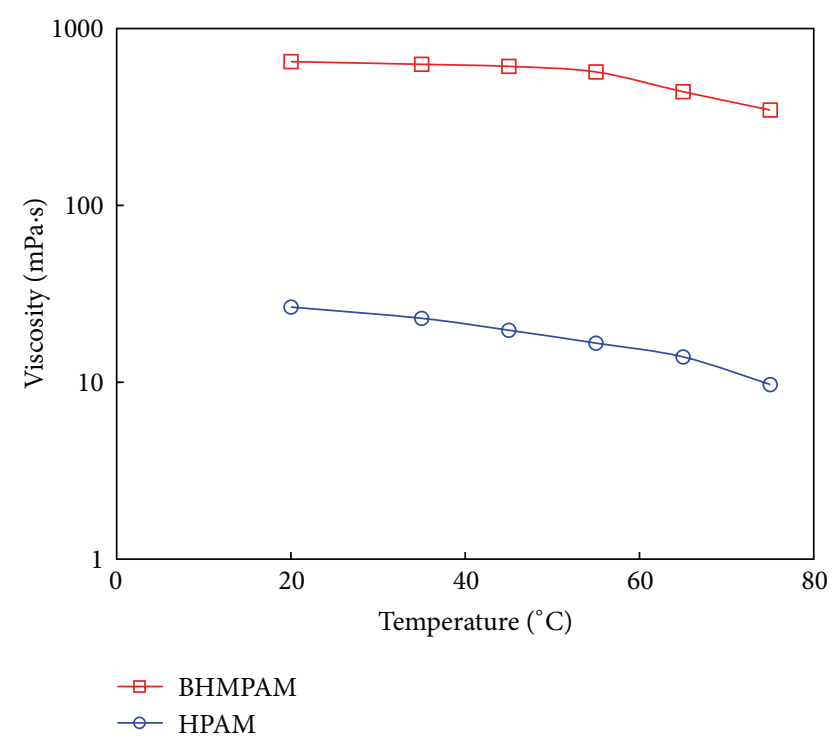

FIGURE 3: Viscosity versus temperature of BHMPAM compared to HPAM in synthetic formation water (concentration of both polymers: $1750 \mathrm{mg} / \mathrm{L}$ ).

When the BHMPAM solution concentration exceeded associating concentration, the hydrophobic association formed mainly between different polymer chains, which generated supramolecular aggregates. The hydrophobic association may present a reversible equilibrium state with

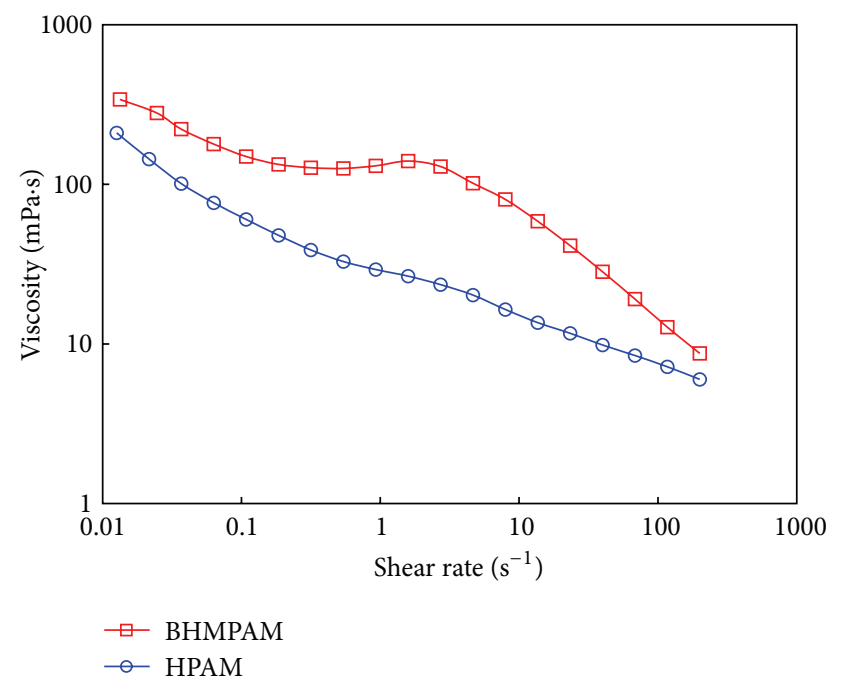

FIGURE 4: Viscosity versus shearing rate of BHMPAM compared to HPAM in synthetic formation water (concentration of both polymers: $1750 \mathrm{mg} / \mathrm{L}$ ).

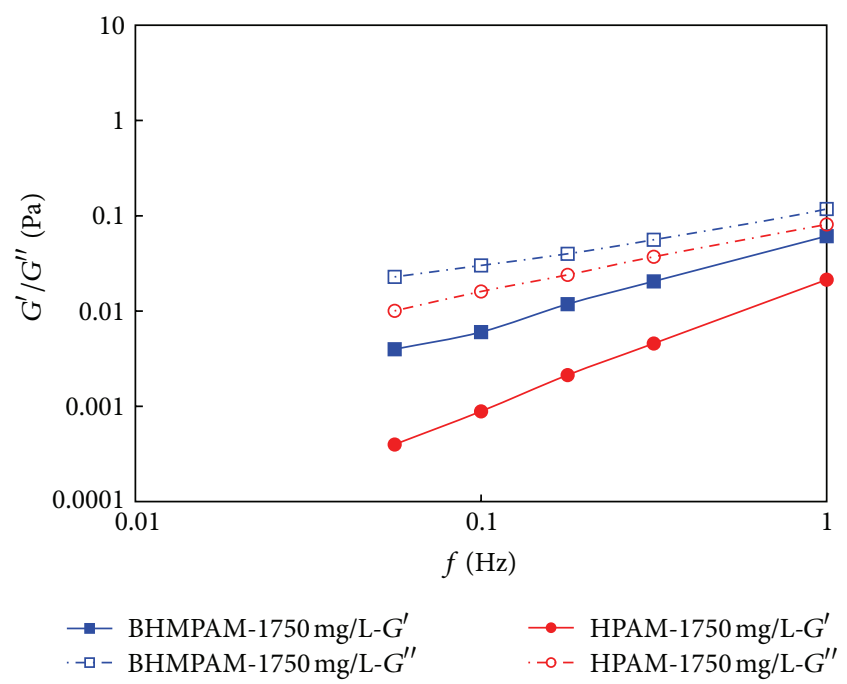

FIGURE 5: Viscoelasticity versus vibrational frequency of BHMPAM compared to HPAM in synthetic formation water (concentration of both polymers: $1750 \mathrm{mg} / \mathrm{L}$ ).

both formation and destruction under shearing. With the increasing of shearing rate, the destruction rate of the association of aggregates was greater than the rate of formation, so that the size of the aggregates in the solution decreased, and the viscosity decreased with the increasing 
of shear rate and the polymer solution performed shear thinning [12].

BHMPAM's viscosifying is superior to HPAM under shearing. On one hand, the hydrophobic association formed network structure in solution; on the other hand, BHMPAM's degree of branching was so high that the hydrodynamic radius was relatively small, while compared to the same molecular weight of straight chain HPAM. The results were absolutely obvious; that is, the entanglement of molecular chains increased and the chain was hard to cut.

3.5. Viscoelasticity. The $G^{\prime}, G^{\prime \prime}$ of BHMPAM and HPAM were unceasingly going up along with the increasing of vibrational frequency which was shown in Figure 5. Because $G^{\prime \prime}$ was larger than $G^{\prime}$, the viscosity of polymer solution was greater than elasticity, and the viscoelasticity of BHMPAM increased dramatically compared with HPAM which was beneficial for BHMPAM as oil displacement agent.

Solution structure was formed by entanglement or intermolecular interaction. The deformation of solution structure would consume energy under imposed stress; at this time, the polymer solution would present viscosity. Also, the deformation of solution structure was a relaxation process, and the elasticity of polymer would be triggered when the deformation lagged behind imposed stress [13]. The viscoelasticity of BHMPAM increased, because the hydrophobic association formed network structure in solution and the high degree of branching increased the entanglement of molecular chains in BHMPAM solution which enhanced the solution structure and extended the relaxation time of deformation.

\section{Conclusion}

(1) A new branched hydrophobically modified polyacrylamid (BHMPAM) was synthesized by the free radical copolymerization; this method is simple and convenient and can be easily implemented into industrial production.

(2) The structure of main chain of BHMPAM is highly branched, which enhances the temperature tolerance and the salinity tolerance of polymer aqueous solution, and BHMPAM shows the property of shearing thinning which is beneficial for injection.

(3) BHMPAM shows higher viscosifying ability, and the viscoelasticity, especially elasticity of BHMPAM, increases dramatically compared with HPAM, which makes BHMPAM able to meet the requirements for oil displacement agent used in high temperature and high salinity reservoir.

\section{Acknowledgment}

This financial support by the special fund of China's central government for the development of local colleges and universities - the project of National First-Level Discipline in Oil and Gas Engineering is gratefully acknowledged.

\section{References}

[1] L. D. Chen, "Polyacrylamides for enhanced oil recoveries," Oilfield Chemistry, vol. 10, no. 3, pp. 283-290, 1993.

[2] D. G. Peiffer, "Hydrophobically associating polymers and their interactions with rod-like micelles," Polymer, vol. 31, no. 12, pp. 2353-2360, 1990.

[3] S. Biggs, A. Hill, J. Selb, and F. Candau, "Copolymerization of acrylamide and a hydrophobic monomer in an aqueous micellar medium: effect of the surfactant on the copolymer microstructure," The Journal of Physical Chemistry, vol. 96, no. 3, pp. 1505-1511, 1992.

[4] S. Zou, W. Zhang, X. Zhang, and B. Jiang, "Study on polymer micelles of hydrophobically modified ethyl hydroxyethyl cellulose using single-molecule force spectroscopy," Langmuir, vol. 17, no. 16, pp. 4799-4808, 2001.

[5] Y. J. Feng, C. Q. Luo, P. Y. Luo et al., "Study on characterization of microstructure of hydrophobically associating watersoluble polymer in aqueous media by scanning electron microscopy and environmental scanning electron microscopy," Acta Petroleli Sinica, vol. 17, no. 6, pp. 39-44, 2001.

[6] C. Gao and D. Yan, "Hyperbranched polymers: from synthesis to applications," Progress in Polymer Science, vol. 29, no. 3, pp. 183-275, 2004.

[7] K. Ishizu, D. Takahashi, and H. Takeda, "Novel synthesis and characterization of hyperbranched polymers," Polymer, vol. 41, no. 16, pp. 6081-6086, 2000.

[8] L. T. Shi, Z. B. Ye, H. Liji, S. X. Wang, and K. L. Zhou, "Preparation method and application of one anti-shearing polymer," China, CN201210117149.9[P], August 152012.

[9] J. Wang, Y. Zheng, Y. J. Feng, and P. Y. Luo, "A novel associative polymer for EOR: performance properties," Oilfield Chemistry, vol. 16, no. 2, pp. 149-152, 1999.

[10] F. S. Hwang and T. E. Hogen-Esch, "Effects of water-soluble spacers on the hydrophobic association of fluorocarbonmodified poly(acrylamide)," Macromolecules, vol. 28, no. 9, pp. 3328-3335, 1995.

[11] X. H. Huang and G. Q. Xu, "Study on solution properties of poly(acrylamide/tetrad-ecyl acrylate) hydrophobically associating copolymer," Fine Chemicals, vol. 17, no. 3, pp. 152-155, 2000.

[12] B. G. Cao, The experiment study on the rheological properties and viscoelastic of hydrophobic associating polymer solution used to displace crude oil [Ph.D. thesis], Southwest Petroleum University, Chengdu, China, 2006.

[13] Y. X. Sun, The research on the mechanism of oil displacement efficiency by polymer flooding with viscoelasticity [Ph.D. thesis], Daqing Petroleum Institute, Daqing, China, 2009. 

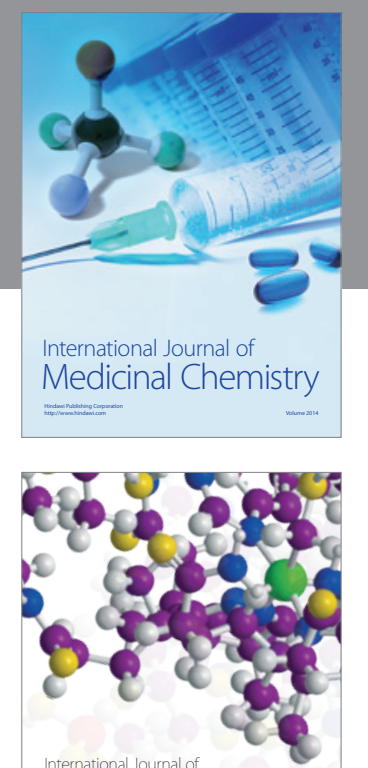

\section{Carbohydrate} Chemistry

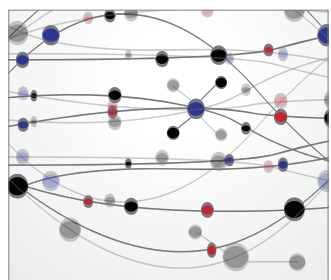

The Scientific World Journal
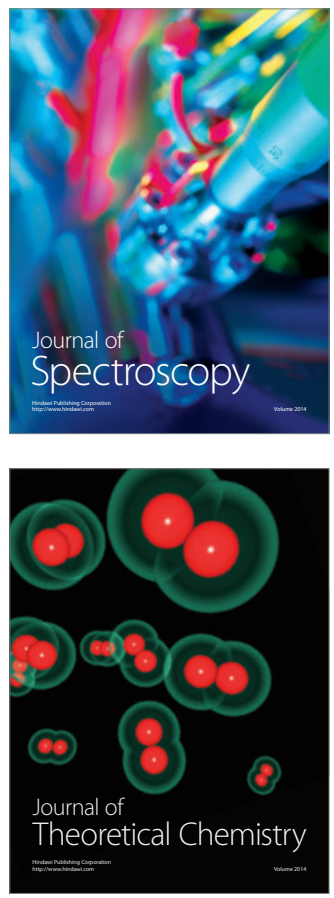
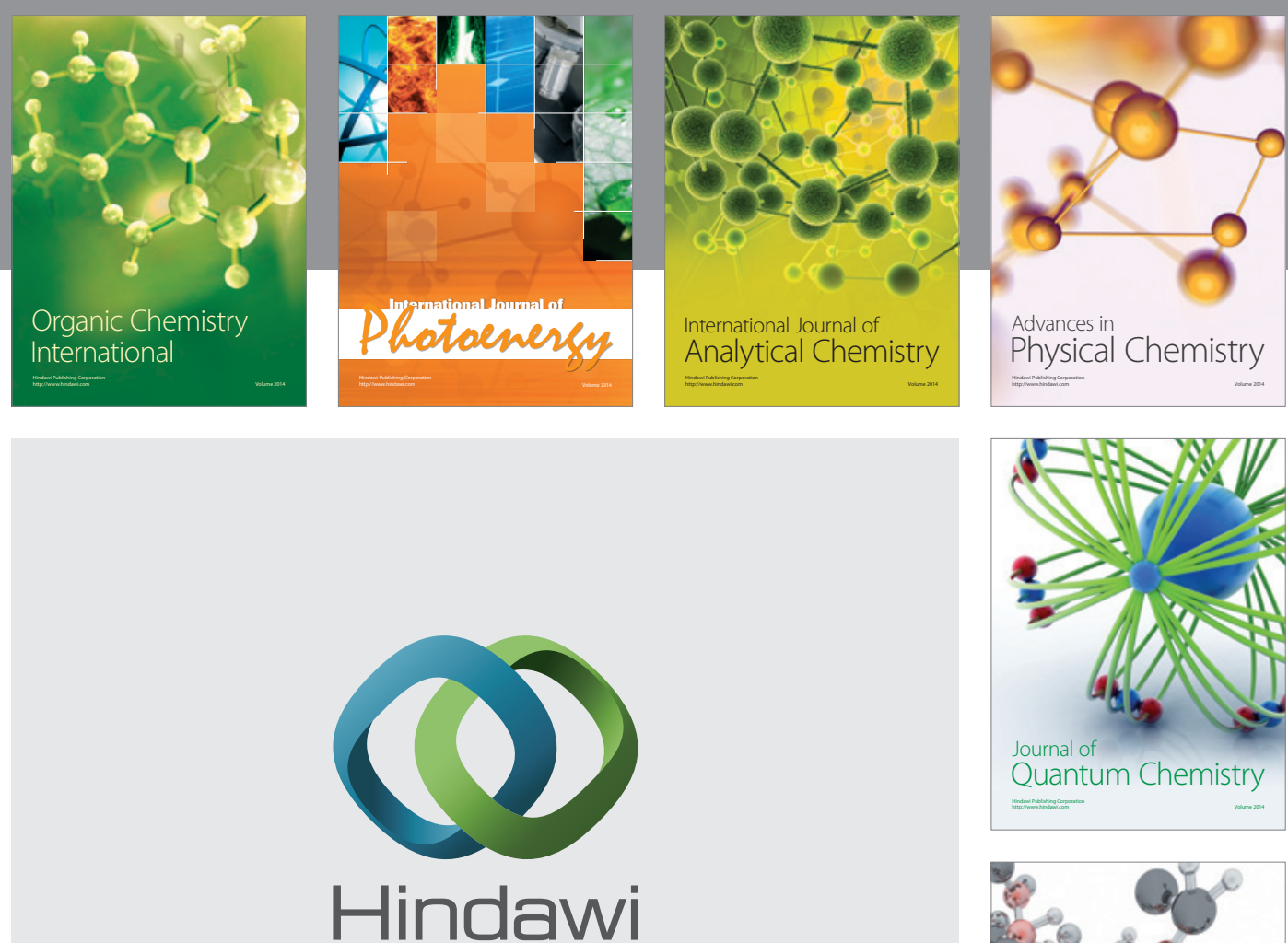

Submit your manuscripts at

http://www.hindawi.com

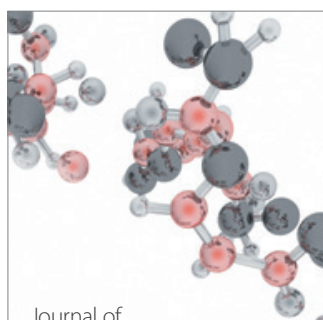

Analytical Methods

in Chemistry

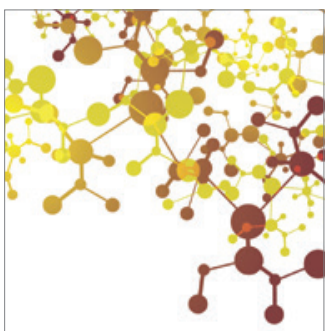

Journal of

Applied Chemistry

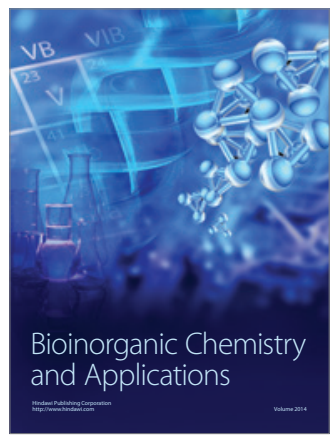

Inorganic Chemistry
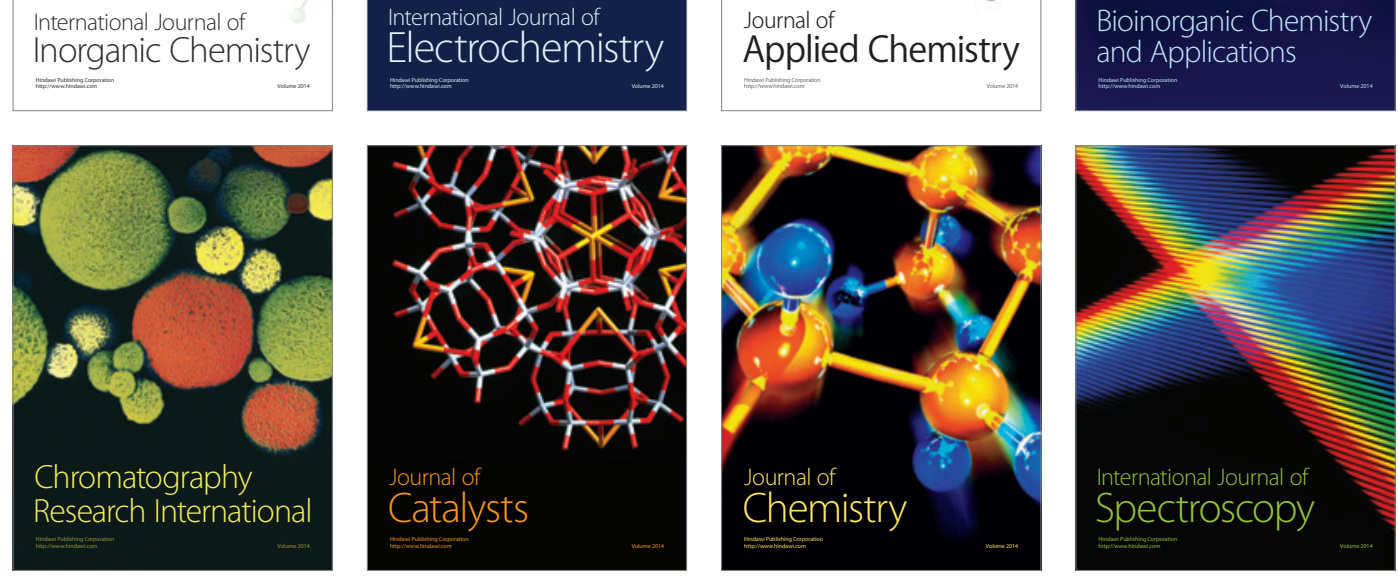